\title{
A novel role of angiopoietin-like-3 associated with podocyte injury
}

Junchao Liu', Xia Gao', Yihui Zhai', Qian Shen', Li Sun', Chun Feng², Jia Rao', Haimei Liu', Xiliang Zha³, Muyi Guo ${ }^{4}$, Duan Ma5, Zhigang Zhang ${ }^{4}$, Ruixi $\mathrm{Li}^{6}$ and Hong $\mathrm{Xu}^{1}$

BACKGROUND: Angiopoietin-like-3 (ANGPTL3) expression is increased in glomerular podocytes of nephrotic syndrome. We hypothesize whether ANGPTL3 plays an important role in podocyte injury and promoting proteinuria.

METHODS: Angpt/3+/+ and Angpt/3-/-female mice on B6;129S5 gene background were injected with adriamycin by tail vein at the dose of $25 \mathrm{mg} / \mathrm{Kg}$ to produce nephropathy. Proteinuira was measured and podocytes ultrastructure was observed by electron microscopy. The interaction between ANGPTL3 and intergrin $\beta 3$ was analyzed by CO-IP and confocal immunofluorescence. The relative gene and protein expression were analyzed by RT-PCR and western blot.

RESULTS: The deletion of ANGPTL3 tremendously attenuates proteinuria (more than a fivefold decrease in albuminuria) and protects podocytes from injury in a mouse model of adriamycininduced nephropathy. We further demonstrate that ANGPTL3 interacts with and activates podocyte-expressed integrin $\beta 3$ and regulate expression of a-actinin-4, which may result in the cytoskeletal rearrangement of podocytes. Additionally, we identify the activation of the ANGPTL3-integrin $\beta 3$ signaling pathway in patients with nephrotic syndrome.

CONCLUSION: ANGPTL3 might play a crucial role in podocyte injury. Either decreasing ANGPTL3 expression or interfering with the ANGPTL3-integrin $\beta 3$ interaction might be benefit for podocyte protection and decrease proteinuira.

$\mathbf{L}^{2}$ ong-term and significant proteinuria is closely related to the progressive decline of kidney function (1). Podocyte injury is believed to be important in promoting proteinuria. Previous studies concerning podocyte biology have revealed the molecular mechanisms behind several genetic disorders (2). Recent studies, however, have explored several circulating or podocytesecreted proteins, such as the serum-soluble urokinase receptor (3), vascular endothelial growth factor (4), and angiopoietinlike-4 (5), which result in damage to the glomerular filtration barrier, probably via an autocrine or paracrine pathway. These findings have attracted the attention of many researchers.
In 2001, using a microarray study, we found that angiopoietin-like-3 (ANGPTL3) expression was significantly increased in the kidney tissue of children with nephrotic syndrome, who characterized with massive proteinuria and diffuse effacement of podocyte foot processes. ANGPTL3 is a secreted protein with a characteristic angiopoietin structure: a signal peptide for secretion, a helical domain that forms coiled coils (CLD), and a C-terminal globular fibrinogen-like domain (FLD) (6). The CLD inhibits lipase activity, leading to elevated plasma triglyceride levels in mice (7), and the FLD induces angiogenesis by binding to integrin $\alpha \mathrm{V} \beta 3$ (8). ANGPTL3 is mainly synthesized by hepatocytes and secreted into the circulation, acting on liver $\mathrm{X}$ receptors in fat cells (9). Under normal conditions, ANGPTL3 is expressed in low amounts in the kidney, and its significance in proteinuria is unknown.

In our previous study, we found upregulation of ANGPTL3 expression not only in glomeruli of children with nephrotic syndrome (10), but also in glomerular podocytes of rats with adriamycin-induced nephropathy (11), and in cultured mouse podocytes treated with adriamycin or puromycin aminonucleoside (PAN) (12,13). Moreover, we found that upregulating podocyte ANGPTL3 expression in vitro stimulates podocyte F-actin rearrangement (14) and increases podocyte motility (13). These experiments indicate that an increased level of ANGPTL3 expression affects podocyte cytoskeleton and function and may be related to promoting proteinuria.

To further clarify whether the altered ANGPTL3 expression in the kidney is merely a satellite phenomenon of proteinuria or an important regulatory factor that influences the disease occurrence and outcome, we investigated the role of ANGPTL3 in Angptl3 ${ }^{-1-}$ mice and established a model of proteinuria in mice. We conducted pathological, cellular, and molecular analyses to answer these questions, and our findings help elaborate the mechanism by which ANGPTL3 regulates the occurrence and development of proteinuria via interacting with and activating integrin $\beta 3$, thus affecting the podocyte cytoskeleton. Finally, this study develops new ideas

\footnotetext{
The first three authors contributed equally to this work.

'Department of Nephrology and Rheumatology, Children's Hospital of Fudan University, Shanghai, China; ${ }^{2}$ Institute of Nutritional Sciences, Shanghai Institutes for Biological Sciences, Chinese Academy of Sciences, Shanghai, China; ${ }^{3}$ Department of Biochemistry and Molecular Biology, Shanghai Medical College of Fudan University, Shanghai, China; ${ }^{4}$ Department of Pathology and Key Laboratory of Molecular Medicine, Shanghai Medical College of Fudan University, Shanghai, China; ${ }^{5}$ Institute of Biomedical Sciences, Fudan University, Shanghai, China; ${ }^{6}$ Department of Anatomy, Histology and Embryology, Shanghai Medical College of Fudan University, Shanghai, China. Correspondence: Hong Xu (hxu@shmu.edu.cn) Received 28 April 2014; accepted 5 November 2014; advance online publication 25 March 2015. doi:10.1038/pr.2015.38
} 
for the in-depth study of molecular-targeted therapy for proteinuria diseases, such as nephrotic syndrome.

\section{RESULTS}

\section{Angpt $/ 3^{-1-}$ Mice Have Phenotypes Similar to Wild-Type Mice}

Except the Presence of Hypolipidemia

Angptl3 ${ }^{+/}$mice were purchased from Mutant Mouse Regional Resource Centers (MMRRC), and Angptl3-- (KO) mice were bred by mating heterozygous mice with heterozygous mice. Genotyping was performed by PCR (Figure 1a). Immunofluorescence confirmed no ANGPTL3 expression in the liver or kidney of KO mice (Figure 1b).

When comparing KO mice and wild-type (WT) mice phenotypes, we found that the KO mice had a body shape that was similar to that of the WT mice as previously reported (15), and there was no difference in their body weights at $8 \mathrm{wk}$ of age (KO $26.02 \pm 0.44 \mathrm{~g}$ vs. WT $25.73 \pm 0.70 \mathrm{~g}, P>0.05$ ). As to the urinary albumin level, testing strips and SDS-PAGE showed an absence of albuminuria in KO mice and WT mice. The plasma albumin level was normal in both groups (KO $29.85 \pm 0.71 \mathrm{~g} / \mathrm{l}$ vs. WT $28.75 \pm 0.41 \mathrm{~g} / 1, P>0.05$ ), while the plasma lipid levels were significantly lower in the KO mice than in the WT mice, as predicted, due to the lipase inhibitory effect of ANGPTL3 (cholesterol: KO $1.82 \pm 0.15 \mathrm{mmol} / \mathrm{l}$ vs. WT $2.86 \pm 0.13 \mathrm{mmol} / \mathrm{l}$,
$P<0.01$; triglyceride $\mathrm{KO} 0.48 \pm 0.02 \mathrm{mmol} / \mathrm{l}$ vs. WT $0.87 \pm 0.12$ $\mathrm{mmol} / \mathrm{l}, P<0.01$ ) (Figure 1c). The glomeruli of the $\mathrm{KO}$ mice stained with PAS showed the same normal structure as the WT mice under light microscopy, and the ultrastructure of the podocytes showed no effacement of foot processes or proliferation of microvilli under transmission electron microscopy (TEM) or scanning electron microscopy (SEM) in either group of mice (Figure 1d).

\section{Adriamycin Induces Podocyte Injury and Proteinuria in WT} Female B6;129S5 Mice at a Higher Dose Than That Required for the Induction of Proteinuria in Rats, and ANGPTL3 Deletion Suppresses the Induction of This Nephropathy Adriamycin is commonly used in mice to induce podocyte injury and proteinuria. However, even at a high dose of adriamycin, proteinuria cannot be induced in C57BL/6J male mice, while it can be induced in the female mice with a $25 \mathrm{mg} / \mathrm{kg}$ dose via tail vein injection, and these mice begin to die at day 5 after injection (16). Therefore, in the present study, we intravenously injected adriamycin at a high dose of $25 \mathrm{mg} / \mathrm{kg}$ and successfully established for the first time an adriamycin-induced podocyte injury and proteinuria model in WT female mice with a B6;129S5 gene background; the affected mice exhibited hypoalbuminemia, massive proteinuria, hyperlipidemia, and

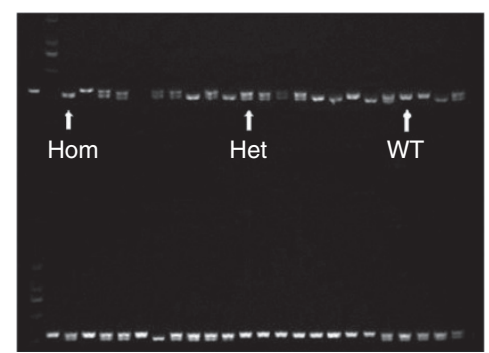

b

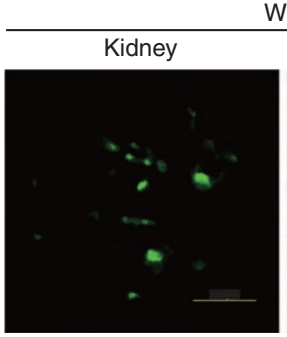

WT
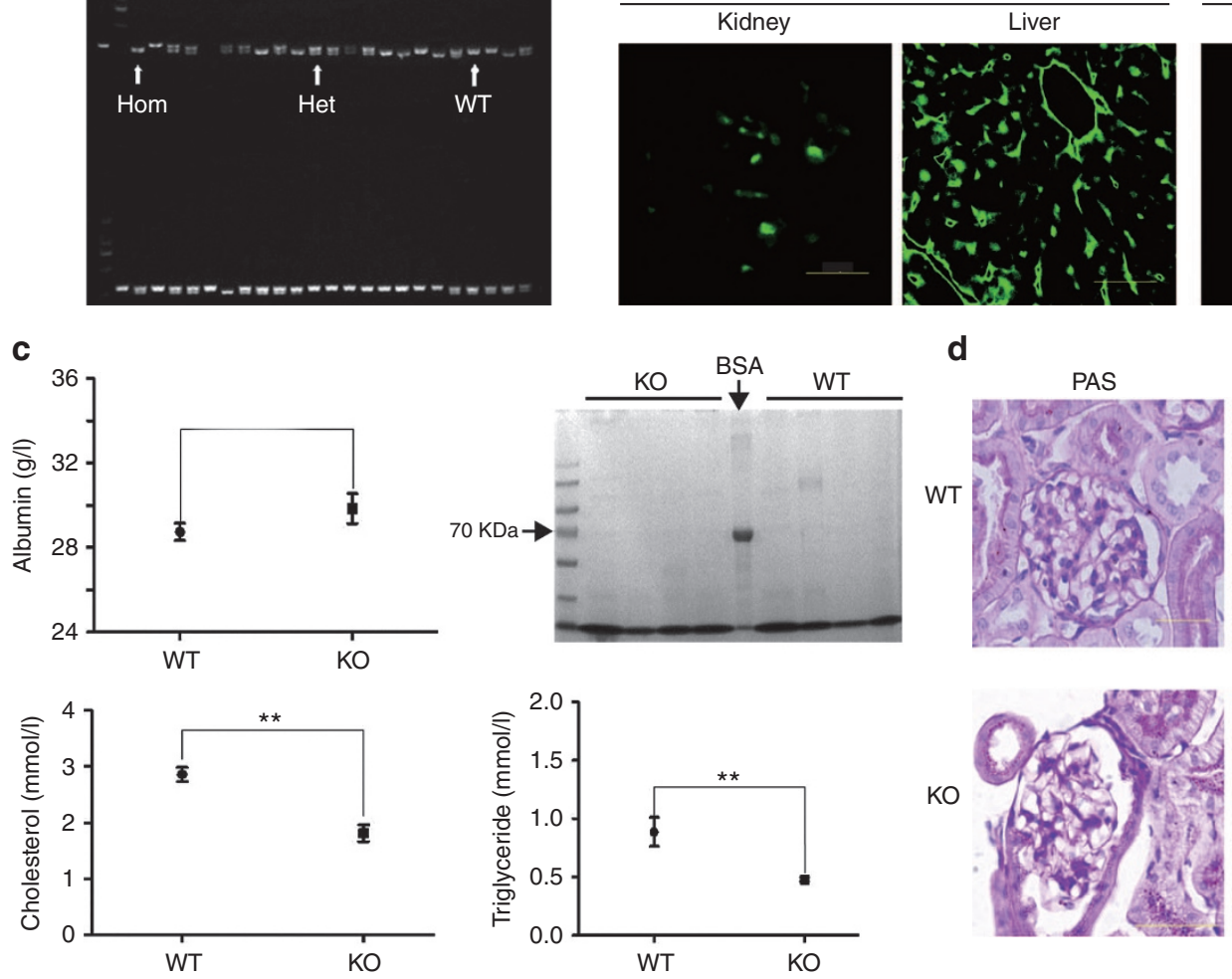

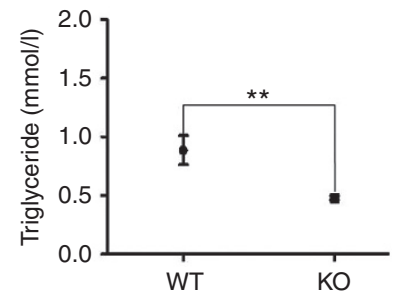

d
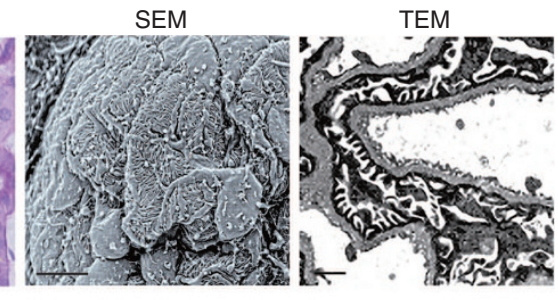

$\mathrm{KO}$
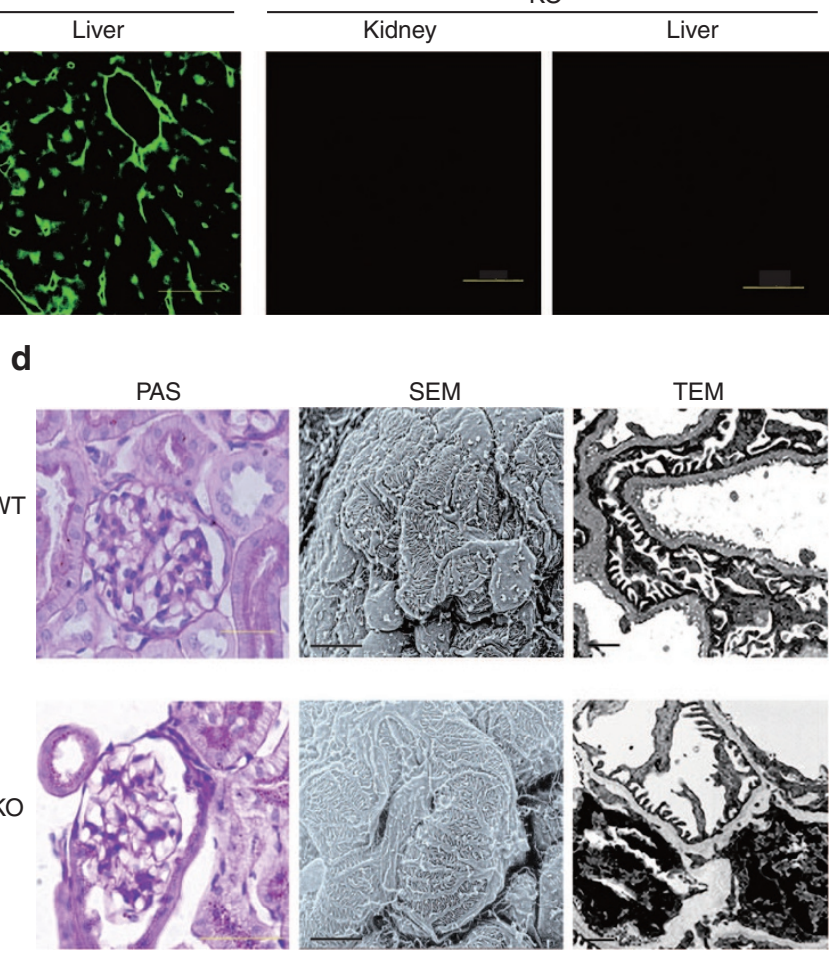

Figure 1. Characterization of Angpt/3-/- (KO) mice. (a) ANGPTL3 deletion in mice was identified by PCR. The band of 308 bp refers to wild-type (WT) mice, $273 \mathrm{bp}$ refers to KO mice, and both bands positive refers to heterozygous mice. (b) ANGPTL3 deletion in mice was identified by immunofluorescence (bar $=100 \mu \mathrm{m}$ ). (c) The plasma albumin level, cholesterol level and triglyceride level of WT mice and KO mice. SDS-PAGE showed albuminuria level in KO mice and WT mice. Bovine serum albumin (BSA) was used as a positive control. ${ }^{*} P<0.01$. (d) The glomeruli of the KO mice and WT mice were stained with PAS $(\times 100$, bar $=100 \mu \mathrm{m})$, and the ultrastructure of the podocytes were examined using transmission electron microscopy $($ TEM, $x 10,000$, bar $=1 \mu \mathrm{m})$ and scanning electron microscopy (SEM, $\times 5,000$, bar $=5 \mu \mathrm{m})$ in two groups of mice. 
the diffuse effacement of foot processes in the podocytes. Six days after the intravenous injection of adriamycin, the plasma albumin levels of the WT mice fell to $23.8 \pm 0.3 \mathrm{~g} / \mathrm{l}$, which was significantly lower than that of the saline-injected WT group $(29.52 \pm 0.79 \mathrm{~g} / \mathrm{l}, P<0.001)$. The urine albumin:creatinine ratio of the adriamycin-injected WT mice was $272.48 \pm 1.84 \mu \mathrm{g} / \mathrm{mg}$, which was significantly higher than that of the saline-injected WT group $(4.11 \pm 0.78 \mu \mathrm{g} / \mathrm{mg}, P<0.0001)$. The plasma cholesterol level of the adriamycin-injected WT mice was $3.37 \pm 0.18$ $\mathrm{mmol} / \mathrm{l}$, which was significantly higher than that of the salineinjected WT group $(2.06 \pm 0.23 \mathrm{mmol} / \mathrm{l}, P<0.005)$. The plasma triglyceride level of the adriamycin-injected WT mice was $1.2 \pm 0.17 \mathrm{mmol} / \mathrm{l}$, which was significantly higher than that of the saline-injected WT group $(0.73 \pm 0.08 \mathrm{mmol} / \mathrm{l}, P<0.005)$ (Figure 2a). In addition, the adriamycin-injected WT mice appeared to be significantly weaker than the saline-injected WT mice, as manifested by significantly sparser hair that was dull and prone to falling out, significantly reduced activity levels, and a curled body position.

Regarding the renal histopathological changes, no difference was found under light microscopy between the adriamycin-injected WT mice and the saline-injected WT mice, as manifested by the clear glomerular structure in both groups, as predicted. However, under TEM and SEM, the appearance of the podocytes between the two groups of mice was significantly different, as manifested by the significant effacement of the podocyte foot processes and the significant podocyte microvillus protrusion in the adriamycin-injected WT mice compared to the saline-injected controls (Figure 2b).

a
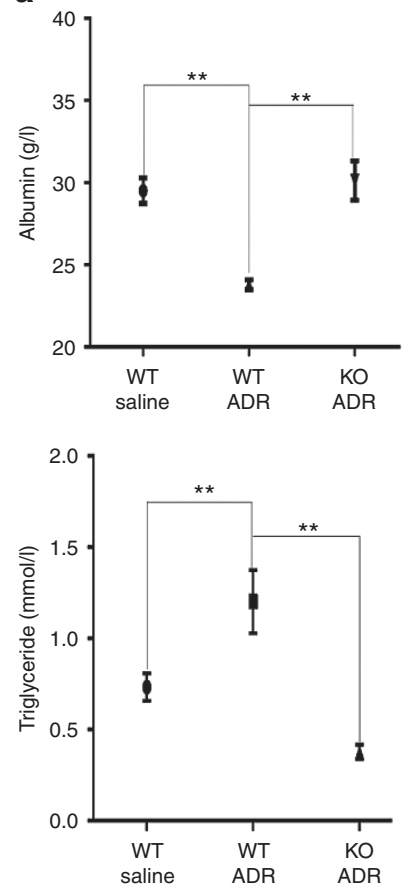
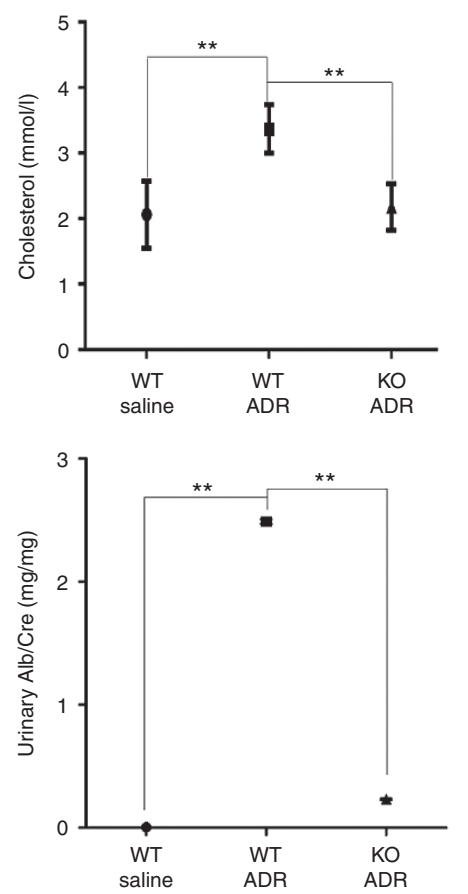

In contrast, the adriamycin-injected $\mathrm{KO}$ mice presented entirely differently compared to the adriamycin-injected WT mice. The appearance of the adriamycin-injected KO mice was similar to the saline-injected WT mice. Interestingly, the adriamycin-injected $\mathrm{KO}$ mice did not show hypoalbuminemia, and their plasma albumin level was normal $(30.14 \pm 1.2 \mathrm{~g} / \mathrm{l})$, which was nearly $30 \%$ higher than that of the adriamycin-injected WT mice $(23.8 \pm 0.3 \mathrm{~g} / \mathrm{l} ; P<0.005)$. The urine albumin:creatinine ratio of the adriamycininjected $\mathrm{KO}$ mice was $50.66 \pm 1.05 \mu \mathrm{g} / \mathrm{mg}$, which was fivefold lower than that of the adriamycin-injected WT mice $(272.48 \pm 1.84 \mu \mathrm{g} / \mathrm{mg} ; P<0.0001)$. The plasma triglyceride level of the adriamycin-injected $\mathrm{KO}$ mice was $0.38 \pm 0.04$ $\mathrm{mmol} / \mathrm{l}$, which was significantly lower than that of the adriamycin-injected WT mice $(1.2 \pm 0.17 \mathrm{mmol} / 1, P<0.005)$. The plasma cholesterol level of the adriamycin-injected KO mice was $2.18 \pm 0.14 \mathrm{mmol} / \mathrm{l}$, which was also significantly lower than that of the adriamycin-injected WT mice (3.37 $\pm 0.18, P<0.005)$ (Figure 2a).

The renal histopathological changes showed no differences under light microscopy (clear glomerular structure) between the adriamycin-injected $\mathrm{KO}$ mice and the adriamycin-injected WT mice. However, TEM and SEM showed significant differences in the podocyte lesions between two groups, as manifested by the significant fusion of podocyte foot processes and significant podocyte microvillus protrusion in the adriamycin-injected WT mice and an almost normal podocyte structure in the adriamycin-injected $\mathrm{KO}$ mice (Figure 2b). b

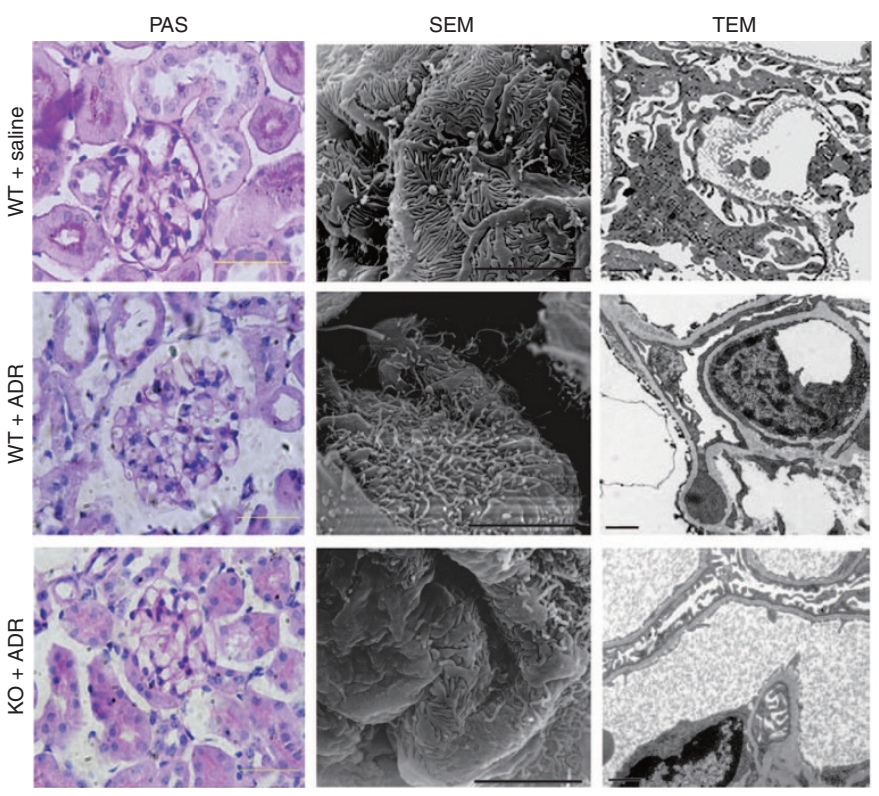

Figure 2. ANGPTL3 deletion suppresses the induction of nephrosis by Adriamycin in WT female B6;129S5 mice. (a) The plasma albumin level, cholesterol level, triglyceride level, and urine albumin:creatinine ratio of WT mice and KO mice with or without ADR injection. ${ }^{* *} P<0.01$. (b) The glomeruli of mice of different groups were stained with PAS $(\times 100$, bar $=100 \mu \mathrm{m})$, and the ultrastructure of the podocytes were examined using TEM $(\times 10,000, \mathrm{bar}=1 \mu \mathrm{m})$ and $\operatorname{SEM}(\times 10,000, \mathrm{bar}=5 \mu \mathrm{m})$. 
ANGPTL3 Interacts With and Activates Integrin $\beta 3$ in Cultured Mouse Podocytes

Camenisch et al. reported that ANGPTL3 can directly bind to integrin $\beta 3$ in human microvascular vein endothelial cells (8). Wei et al. reported that integrin $\beta 3$ plays an important role in proteinuria production, interfering with podocyte cytoskeleton rearrangement (17). In our previous study, we found that integrin $\alpha V \beta 3$ mediated F-Actin rearrangement through small GTPases Rac1 and RhoA, and integrin-mediated FAK/PI3K phosphorylation is important for Rac1 activation and podocytes lamellipodia formation (14). Here, we hypothesized that the podocyte-secreted ANGPTL3 binds to and activates integrin $\beta 3$ on the podocytes themselves, thereby inducing podocyte cytoskeletal rearrangement and regulating podocyte motility via this autocrine or paracrine pathway, promoting proteinuria.

To test this hypothesis, we firstly performed a confocal microscopy study and demonstrated the colocalization of ANGPTL3 and integrin $\beta 3$ in cultured podocytes overexpressing ANGPTL3 (Figure 3a). Coimmunoprecipitation (co-IP) showed that ANGPTL3 and integrin $\beta 3$ formed a complex in cultured podocytes, indicating that ANGPTL3 binds to integrin $\beta 3$ (Figure $3 \mathbf{b}$ ). Furthermore, we investigated whether ANGPTL3 activates integrin $\beta 3$ in podocytes using AP5, a monoclonal antibody that specifically detects the active form of integrin $\beta 3$ (18). The fluorescence-activated cell sorting (FACS) results showed that ANGPTL3 over-expression a
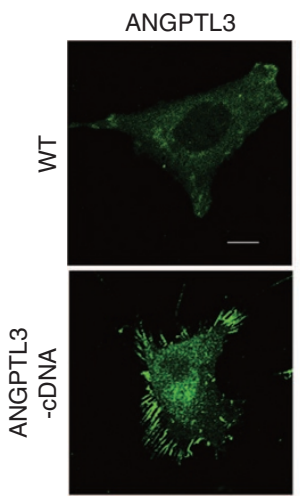
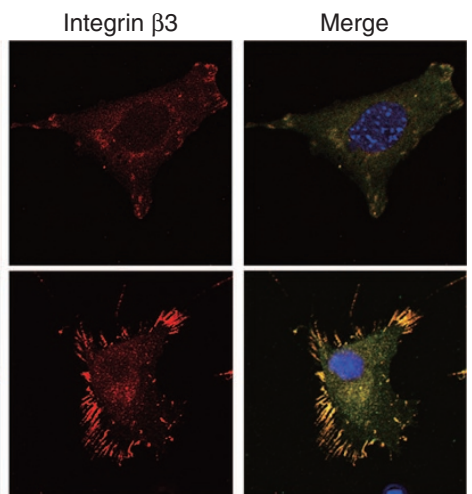

b

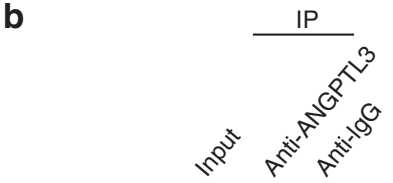

WB:Integrin $\beta 3$

WB: ANGPTL3

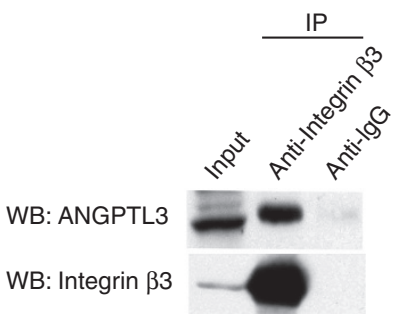

Figure 3. ANGPTL3 interacts with integrin $\beta 3$ in mouse podocytes.

(a) Confocal microscopy images of integrin $\beta 3$ (red) and ANGPTL3 (green) in both wild-type and over-expressing ANGPTL3 podocytes. The merged image (yellow) shows the colocalization of ANGPTL3 and integrin $\beta 3$ in podocytes. Magnifications: $200 \times$. bar $=10 \mu \mathrm{m}$. (b) Podocytes were transfected with plasmid encoding ANGPTL3, and cell lysates were immunoprecipitated with antibodies binding to integrin $\beta 3$ or ANGPTL3. The bound fraction was analyzed using SDS-polyacrylamide gel. significantly increased the percentage of AP5 positive podocytes $(P<0.01)$. Meanwhile, ANGPTL3 knockdown significantly suppressed the increased AP5-positive cell percentage after treatment with adriamycin $(P<0.05$, Figure 4a). These results confirmed our hypothesis that ANGPTL3 interacts with and activates integrin $\beta 3$ in podocytes. We also found colocalization of the ANGPTL3-AP5 complex in the lamellipodia of adriamycin-treated podocytes (Figure $4 \mathrm{~b}$ ), which may indicate that ANGPTL3 is concentrated in the effaced podocyte foot processes, as we also confirmed in the podocytes of adriamycin-induced rats by immunogold staining (Figure 5).

\section{ANGPTL3 Regulates $\alpha$-Actinin-4 Expression in Cultured Mouse Podocytes}

Actin filaments are important for maintaining normal podocyte morphology and motility (19). We previously reported

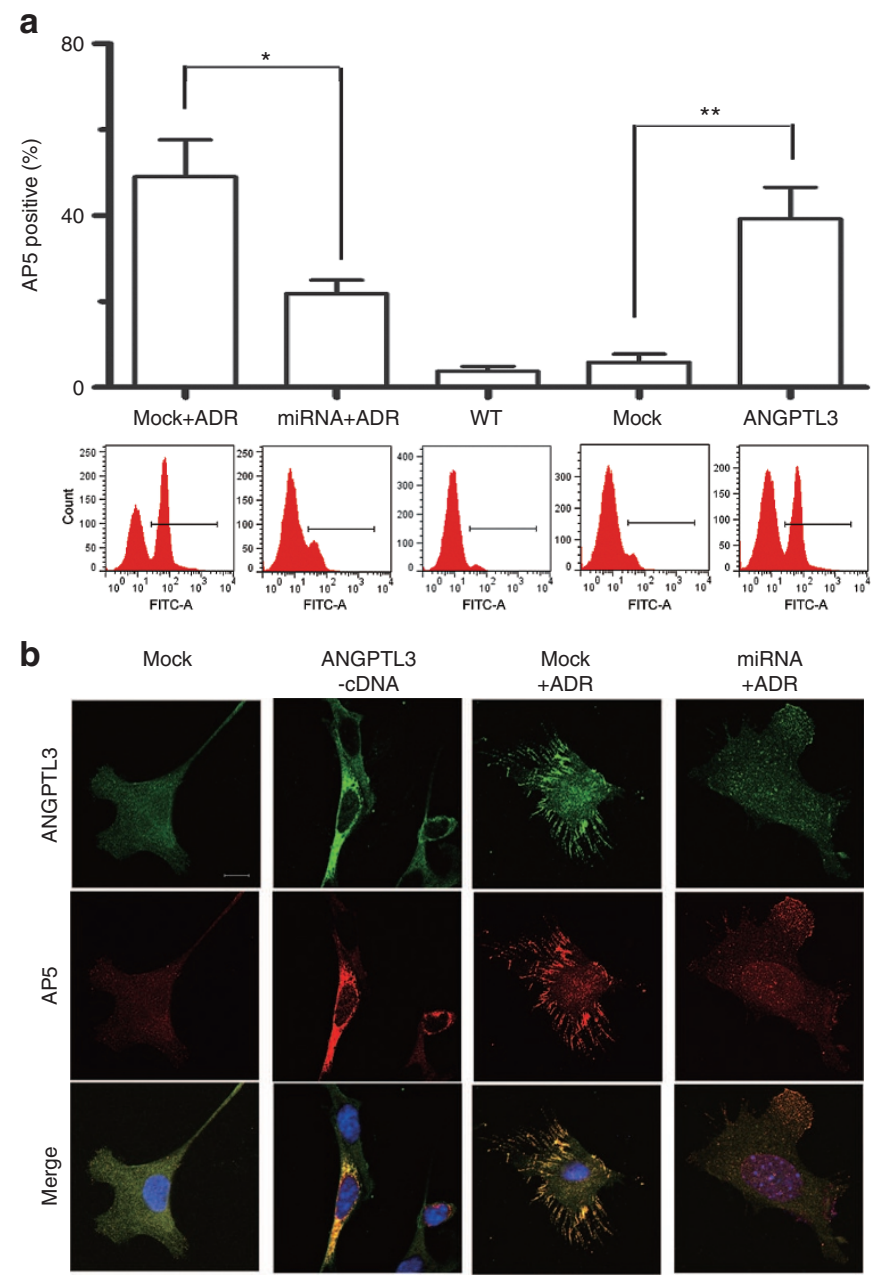

Figure 4. ANGPTL3 activates integrin $\beta 3$ in mouse podocytes. (a) FACS for AP5 showed that the over-expression of ANGPTL3 significantly enhanced the percentage of AP5-positive podocytes, and ADR treatment has a similar capacity to activate AP5 in cultured podocytes. Knockdown of ANGPTL3 powerfully inhibited the AP5 increase in ADR-treated podocytes. (b) Overexpression of ANGPTL3 or treated with ADR, the podocytes formed more spikes and lamellipodia. The confocal image shows that ANGPTL3 (green) colocalizes with AP5 (red), and the subcellular distribution is located preferentially in the lamellipodia of the mouse podocytes. Magnifications: $200 \times$. bar $=10 \mu \mathrm{m}$. ${ }^{*} P<0.05,{ }^{*} P<0.01$. 

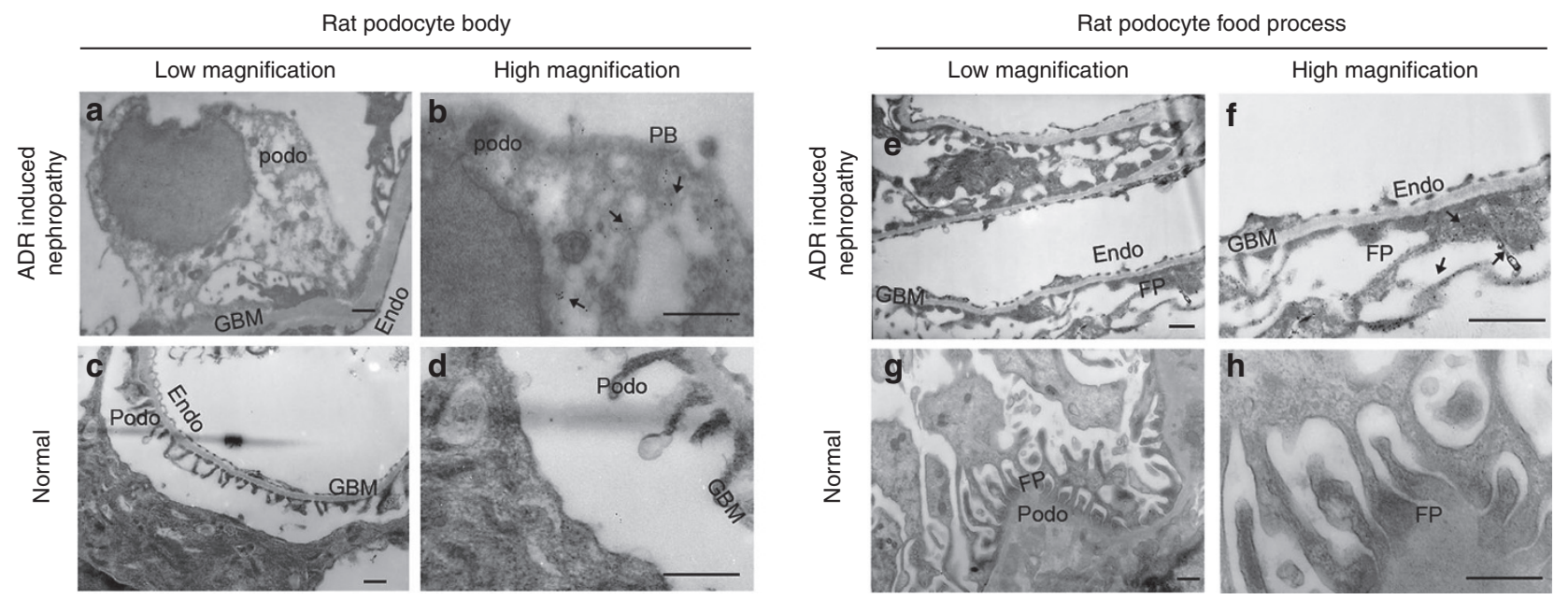

Figure 5. ANGPTL3 is concentrated in the effaced podocyte foot processes. Electron microscopy analyses of colloidal immunogold staining in the ADR-treated glomerulus showed a distribution of ANGPTL3-labeled gold particles mainly in the podocyte cytoplasm rather than in the endothelial cells. The sub cellular localization showed that ANGPTL3 expression was mainly concentrated within the podocyte foot processes. Endo, endothelial; FP, foot process; GBM, glomerular basement membrane; PB, podocyte body; Podo, podocyte. Magnifications: $(\mathbf{a}, \mathbf{c}, \mathbf{g}) \times 9,000,(\mathbf{b}, \mathbf{d}, \mathbf{f}, \mathbf{h}) \times 30,000,(\mathbf{e}) \times 10,000$. Data are representative of five independent experiments and are shown as the mean \pm SEM. (bar $=1 \mu \mathrm{m})$

that ANGPTL3 induces podocyte F-actin rearrangement (14). Alpha-actinin-4, an actin-modulating protein, can cross-link with F-actin to maintain the necessary polarity for actin bundle formation and is a downstream signal for integrin $\beta 3(20-22)$. Recent reports have also shown that either the upregulation or absence of $\alpha$-actinin- 4 in podocytes can lead to renal disease. Therefore, we further investigated the association between ANGTPL3 and $\alpha$-actinin- 4 in vitro. We found that ANGPTL3 significantly affected the mRNA and protein expression of $\alpha$-actinin-4 (Figure 6), suggesting that ANGPTL3 may induce podocyte actin rearrangement by affecting $\alpha$-actinin- 4 expression. Additionally, the ANGPTL3 knockdown substantially inhibited the change of expression of $\alpha$-actinin- 4 for $24 \mathrm{~h}$ after adriamycin treatment (Figure 6). These data indicate that $\alpha$-actinin- 4 expression is affected in response to adriamycin in an ANGPTL3-dependent manner.

Taken together, our findings indicate that ANGPTL3 significantly affects podocyte cytoskeleton dynamics and plays an important role in actin rearrangement.

\section{Activation of the ANGPTL3-Integrin $\beta 3$ Signaling Pathway in Patients With Nephrotic Syndrome}

In vitro, we have found increased expression of ANGPTL3 activates integrin $\beta 3$ and its downstream signaling pathway, leading to cytoskeleton rearrangement of podocytes. In mouse proteinuria model, we have demonstrated that deletion of ANGPTL3 has a protective effect on podocytes cytoskeleton and can reduce proteinuria. Therefore, we further investigated whether this interaction happened in human as well. Renal biopsy tissues from 30 patients with nephrotic syndrome (MCD, $n=16$; FSGS, $n=7$; IgA nephropathy, $n=4$; and membrane nephropathy, $n=3$ ) who presented with massive proteinuria and diffuse effacement of podocyte foot processes were studied. They were from 17 males and 13 females with an average age of $34.1 \pm 15.9 \mathrm{y}$. We found an increased expression of
ANGPTL3, integrin $\beta 3$, and AP5 with confocal microscopy in all samples (Figure 7), indicating that the ANGPTL3-induced activation of integrin $\beta 3$ is involved in the development of foot process effacement and the rearrangement of the cytoskeleton in podocytes and may, thus, accelerate the development of proteinuria in patients with nephrotic syndrome.

\section{DISCUSSION}

In this report, we demonstrate in vivo a novel role for ANGPTL3 in podocyte injury and proteinuria. We found that the deletion of ANGPTL3 greatly attenuates proteinuria and podocyte injury in adriamycin-injected mice. Our in vitro studies indicate that ANGPTL3 can interact with and activate integrin $\beta 3$ and its downstream signaling pathway and may therefore induce the rearrangement of the podocyte cytoskeleton by interfering with $\alpha$-actinin-4. Furthermore, we demonstrated that the ANGPTL3 pathway is activated in the podocytes of patients with proteinuria disease such as nephrotic syndrome.

Our earlier studies revealed the increased expression of ANGPTL3 in the podocytes of children with nephrotic syndrome and of rats with adriamycin-induced nephropathy, as well as in adriamycin- or PAN-treated cultured podocytes. We have identified ANGPTL3-dependent regulation of podocyte motility in vitro $(12,13)$. In this study, our in vivo experiments indicate that the deletion of ANGPTL3 can tremendously reduce proteinuria and preserve podocyte structure and function in adriamycin-injected mice, suggesting that ANGPTL3 can significantly affect the development of proteinuria by interfering with the podocyte cytoskeleton. Human studies have also supported this finding, as there is increased ANGPTL3 expression in the glomeruli of patients with diffuse effacement of foot processes in podocytes.

ANGPTL3 has been reported to directly bind to integrin $\beta 3$ in human microvascular vein endothelial cells (8), and integrin $\beta 3$ activation in podocytes can promote the effacement of 
a

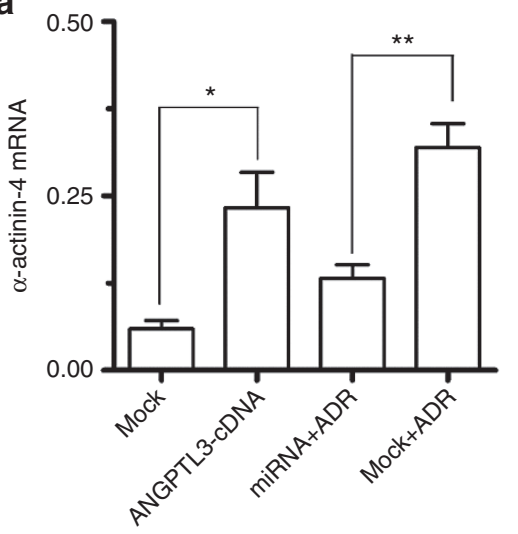

b

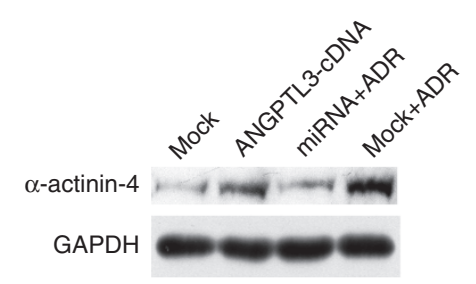

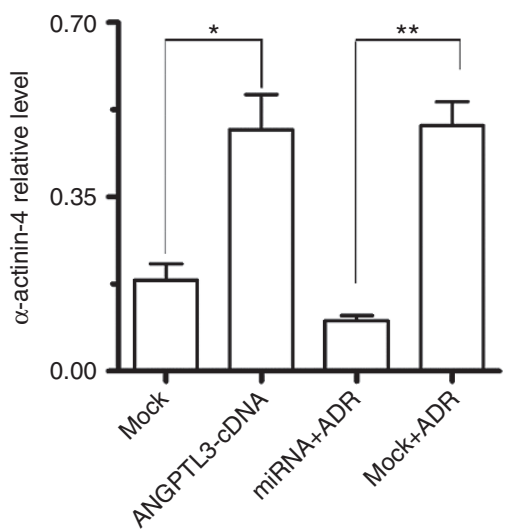

Figure 6. ANGPTL3 regulates $\alpha$-actinin-4 expression in mouse podocytes. (a) The quantitative real-time PCR data for the mRNA levels of $\alpha$-actinin-4 in podocytes. (b) Western blot analysis of $\alpha$-actinin- 4 expression in podocytes that received different treatments. Quantifications of the bands are shown in the right panels. Data are representative of five independent experiments and are shown as the mean $\pm \mathrm{SEM}$. ${ }^{*} P<0.05,{ }^{* *} P<0.01$.

a

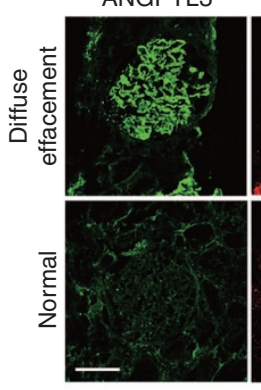

Integrin $\beta 3$

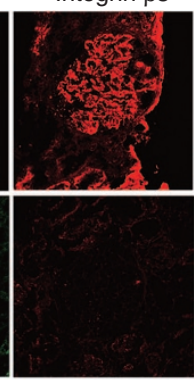

b
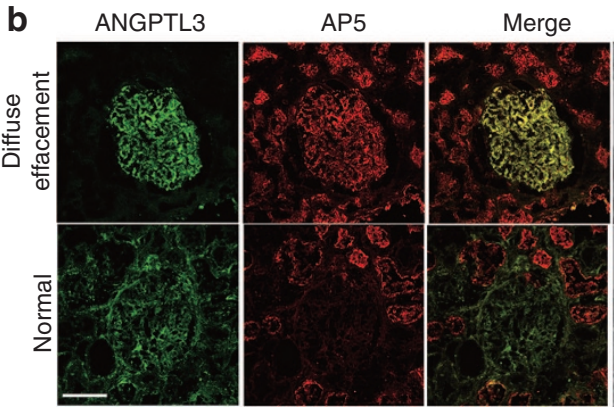

ECM

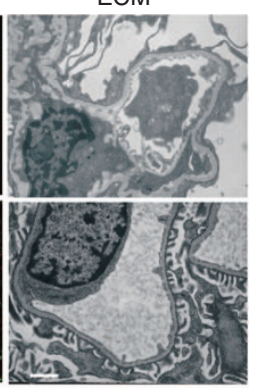

ECM

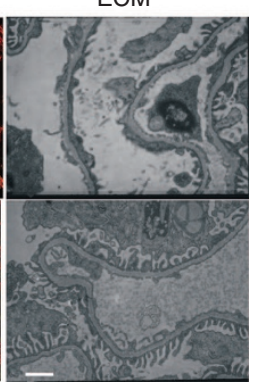

Figure 7. Activation of the ANGPTL3-integrin $\beta 3$ signaling pathway in patients with NS. (a) The expression of ANGPTL3 (green) and integrin $\beta 3$ (red) in renal biopsy tissue of a patient with nephrotic syndrome with diffuse effacement of podocyte foot processes were examined using confocal immunofluorescence microscopy. (b) The expression of ANGPTL3 (green) and AP5 (red) in renal biopsy tissue of a patient with nephrotic syndrome with diffuse effacement of podocyte foot processes were examined using confocal immunofluorescence microscopy. Representative images of one 9-y-old boy with nephrotic syndrome (MCD) and a normal control are shown. bar $=100 \mu \mathrm{m}$ for confocal immunofluorescence image and $500 \mathrm{~nm}$ for ECM.

podocyte foot processes $(3,17)$ and promote proteinuria. We previously further demonstrated integrin $\beta 3$ mediated F-actin rearrangement through FAK/PI3K signaling pathway, causing particular lamellipodia formation in podocytes (14). In this study, we demonstrated that ANGPTL3 changing podocyte morphology by interacting with and activating integrin $\beta 3$ in podocytes, which was proven by the increased detection

of AP5, a monoclonal antibody that specifically binds to the active form of integrin $\beta 3$. Our experiments also indicated that ANGPTL3 regulates $\alpha$-actinin- 4 expression, involved in affecting podocyte cytoskeleton dynamics. Additionally, in human tissue, we also observed a correlation between the diffuse effacement of the foot processes and activation of the ANGPTL3-integrin $\beta 3$ signaling pathway. All these results indicate that activation of the ANGPTL3 pathway in podocytes may induce cytoskeleton rearrangement and result in the effacement of foot processes and proteinuria.

It is of note that Clement et al. recently reported that podocytes secreting ANGPTL4 play a vital role in nephrotic syndrome, possibly due to the reduced sialylation level of ANGPTL4 (5). It will be very interesting to further study the relationship between ANGPTL3 and ANGPTL4 in nephrotic syndrome.

In summary, we report a novel role for ANGPTL3 in podocyte injury regarding proteinuria and describe a novel mechanism for ANGPTL3, wherein ANGPTL3 is involved in the production of proteinuria by triggering integrin $\beta 3$ and the downstream signaling pathway in podocytes; this may result in cytoskeletal rearrangement and the effacement of foot processes. This research helps to deepen the understanding of the potential involvement of angiopoietin-like proteins in proteinuria diseases and lays the foundation for further indepth research into the renoprotective effect of the Angptl3 knockout and the development of a molecular-targeted treatment of podocytes.

\section{METHODS}

\section{Antibodies and Reagents}

The antibodies and reagents used in this study are listed with their sources as follows: AP5 monoclonal antibody against active $\beta 3$ integrin (Genetic Testing Institute, Waukesha, WI); polyclonal antibody against $\alpha$-actinin-4 (Chemicon, Temecula, CA); monoclonal antibody against glyceraldehyde-phosphate dehydrogenase (GAPDH) (Santa Cruz Biotechnology, Dallas, TX); polyclonal antibody against ANGPTL3 (R\&D Systems, Minneapolis, MN); fluorescein phalloidin for labeling F-actin (Invitrogen, Carlsbad, CA); and polyclonal antibody against synaptopodin (Sigma-Aldrich, St Louis, MO). Adriamycin was purchased from Pfizer (New York, NY). 


\section{Angpt/3-/- Mice}

Angptl3 $^{+/-}$mice (B6;129S5-Angptl3tm1Lex, No. 032146-UCD) were purchased from Mutant Mouse Regional Resource Centers (MMRRC) (University of California, Davis, CA). The Angptl3 ${ }^{-1-}$ mice were bred with 2 Angptl $3^{+/-}$mice. The genotypes were identified by PCR, with primers designed by Lexicon Genetics (The Woodlands, TX), (Primers for wild type: 5'-TAG CCT ACT GCT ATG TCA GAC TGC-3' ', 5'-GGG AAG CTA GTG AGA TAA TGA AAG GC-3'; primers for mutation: 5'-TGA TCC CAG ATT TGC GTG AAT AAC C-3', 5'-GCA GCG CAT CGC CTT CTA TC-3').

Establishment of Adriamycin-Induced Nephropathy Model

The animal experiments in this study were approved by the Ethics Committee of Children's Hospital of Fudan University (2012100). The mice and rats were given standard laboratory food and free access to water.

Female B6;129S5 mice at the age of $8 \mathrm{wk}$ received intravenous adriamycin $(25 \mathrm{mg} / \mathrm{kg})$ dissolved in saline at a concentration of $5 \mu \mathrm{g} / \mathrm{ul}$ on day 0 , in accordance with the protocol reported by Jeansson et al. (16). The control group received an identical volume of intravenous saline. There were six mice in each group. From day 0 to 5 , the mice with adriamycin injections were given two daily intraperitoneal injections of $2 \mathrm{ml}$ of a glucose-electrolyte solution to prevent weight loss due to low appetite (16). On day 6, spot-urine and blood were collected for biochemical study, the mice were sacrificed, and the kidneys were removed after saline perfusion for histological study.

Male Sprague-Dawley (SD) rats weighing 95-105 g received intravenous adriamycin $(7 \mathrm{mg} / \mathrm{kg})$ dissolved in $1 \mathrm{ml}$ saline on day 0 . The control group received an identical volume of intravenous saline. Each week, an assay for $24 \mathrm{~h}$ urine protein excretion was performed. Renal samples for immuno-EM were obtained after euthanasia on the 28 th day.

\section{Podocyte Culture and Treatment}

The cultured, immortal mouse podocytes originated in the laboratory of Dr P Mundel in the USA. The management of the cultures was performed according to the standards outlined in Dr Mundel's review (23). The stable expression of nephrin produced by the podocytes was confirmed by western blot analysis.

The cells were $70-80 \%$ confluent prior to ADR or PAN treatment. When the podocytes were well differentiated $14 \mathrm{~d}$ later, ADR or PAN stock solution was added to each well to yield a final concentration of $0.5 \mu \mathrm{mol} / \mathrm{l}(\mathrm{ADR})$ or $50 \mu \mathrm{g} / \mathrm{ml}$ (PAN). The cells were then cultured for another $24 \mathrm{~h}$ and harvested for the subsequent assays. For transfection, the differentiated podocytes were trypsinized and plated in sixwell plates $24 \mathrm{~h}$ prior to transfection in RPMI-1640 containing $10 \%$ fetal bovine serum. Four micrograms of DNA was transfected with 10 $\mu$ l Lipofectamine 2000 reagent (Invitrogen, Carlsbad, CA).

\section{Human Kidney Tissues}

Normal human renal cortical tissue was obtained from five patients undergoing nephrectomies for renal tumors, including three males and two females with an average age of $51.7 \pm 9.2$. Renal biopsies $(n=30)$ were from 30 patients with nephrotic syndrome, including $\operatorname{MCD}(n=16)$, FSGS $(n=7)$, IgA nephropathy $(n=4)$, and membrane nephropathy $(n=3)$; there were 17 males and 13 females with an average age of $34.1 \pm 15.9 \mathrm{y}$. The procedures were in accordance with the local ethical standards of the responsible committee on human experimentation and patient written informed consents were obtained.

\section{Statistical Analysis}

The values, expressed as the means \pm SEM, were subjected to oneway analysis of variance tests, and the Pearson correlations among the groups were calculated. $P$ values of $<0.05$ were considered to be statistically significant.

\section{Laboratory Methods}

See the Supplementary Methods online section for other detailed experimental procedures, including urine protein excretion assay, plasma biochemistry analysis, periodic acid Schiff (PAS) histostain, electron microscopy, plasmids construction, gene transient transfection, quantitative real-time PCR assays, western blot, coimmunoprecipitation, double immunofluorescence labeling, and colloidal gold immune-electron microscopy.

\section{SUPPLEMENTARY MATERIAL}

Supplementary material is linked to the online version of the paper at http:// www.nature.com/pr

\section{ACKNOWLEDGEMENTS}

We thank Zhihui Min (Shanghai Institutes for Biological Sciences, Chinese Academy of Sciences) for technical assistance in fluorescence activating cell sorter (FACS).

\section{STATEMENT OF FINANCIAL SUPPORT}

This work was supported in part by the National Nature Science Foundation of China (30971375), Ministry of Education, Science and Technology Development Center (20110071110071), Science and Technology Commission of Shanghai Municipality (10XD1400700), and a grant from Shanghai Medical College of Fudan University (10L-42).

Disclosure: There is no conflict of interest to disclose.

\section{REFERENCES}

1. Iseki K, Ikemiya Y, Iseki C, Takishita S. Proteinuria and the risk of developing end-stage renal disease. Kidney Int 2003;63:1468-74.

2. Tryggvason K, Patrakka J, Wartiovaara J. Hereditary proteinuria syndromes and mechanisms of proteinuria. N Engl J Med 2006;354: $1387-401$.

3. Wei C, El Hindi S, Li J, et al. Circulating urokinase receptor as a cause of focal segmental glomerulosclerosis. Nat Med 2011;17:952-60.

4. Sison K, Eremina V, Baelde H, et al. Glomerular structure and function require paracrine, not autocrine, VEGF-VEGFR-2 signaling. J Am Soc Nephrol 2010;21:1691-701.

5. Clement LC, Avila-Casado C, Macé C, et al. Podocyte-secreted angiopoietin-like-4 mediates proteinuria in glucocorticoid-sensitive nephrotic syndrome. Nat Med 2011;17:117-22.

6. Ono M, Shimizugawa T, Shimamura M, et al. Protein region important for regulation of lipid metabolism in angiopoietin-like 3 (ANGPTL3): ANGPTL3 is cleaved and activated in vivo. J Biol Chem 2003;278:41804-9.

7. Koishi R, Ando Y, Ono M, et al. Angptl3 regulates lipid metabolism in mice. Nat Genet 2002;30:151-7.

8. Camenisch G, Pisabarro MT, Sherman D, et al. ANGPTL3 stimulates endothelial cell adhesion and migration via integrin alpha vbeta 3 and induces blood vessel formation in vivo. J Biol Chem 2002;277: 17281-90.

9. Conklin D, Gilbertson D, Taft DW, et al. Identification of a mammalian angiopoietin-related protein expressed specifically in liver. Genomics 1999;62:477-82.

10. Rao J, Xu H, Sun L, Zhao ZH, Zhang XR. Expression of ANGPTL3 in children with primary nephrotic syndrome. Chin J Nephrol 2006;22:5.

11. Wu JW, Xu H, Wu Y, Zhu LW, Chen L. Expression of ANGPTL3 in kidney and its relationship to proteinuria and hyperlipidemia in Adriamycininduced nephrotic rats. Chin J Nephrol 2005;21:6.

12. Jia R, Hong X, Li S, Haichun Y, Chuanming H. Expression of angiopoietinlike 3 associated with puromycin-induced podocyte damage. Nephron Exp Nephrol 2010;115:e38-45.

13. Gao X, Xu H, Liu H, Rao J, Li Y, Zha X. Angiopoietin-like protein 3 regulates the motility and permeability of podocytes by altering nephrin expression in vitro. Biochem Biophys Res Commun 2010;399:31-6.

14. Lin Y, Rao J, Zha XL, Xu H. Angiopoietin-like 3 induces podocyte F-actin rearrangement through integrin $\mathrm{a}(\mathrm{V}) \mathrm{B} 3 / \mathrm{FAK} / \mathrm{PI} 3 \mathrm{~K}$ pathway-mediated Rac1 activation. Biomed Res Int 2013;2013:135608.

15. Tang T, Li L, Tang J, et al. A mouse knockout library for secreted and transmembrane proteins. Nat Biotechnol 2010;28:749-55.

16. Jeansson M, Björck K, Tenstad O, Haraldsson B. Adriamycin alters glomerular endothelium to induce proteinuria. J Am Soc Nephrol 2009;20: $114-22$.

17. Wei C, Möller CC, Altintas MM, et al. Modification of kidney barrier function by the urokinase receptor. Nat Med 2008;14:55-63.

18. Honda S, Tomiyama Y, Pelletier AJ, et al. Topography of ligand-induced binding sites, including a novel cation-sensitive epitope (AP5) at the amino terminus, of the human integrin beta 3 subunit. J Biol Chem 1995;270: 11947-54. 


\section{Angptl3-KO attenuates podocyte injury}

Articles

19. Welsh GI, Saleem MA. The podocyte cytoskeleton-key to a functioning glomerulus in health and disease. Nat Rev Nephrol 2012;8:14-21.

20. Dandapani SV, Sugimoto H, Matthews BD, et al. Alpha-actinin-4 is required for normal podocyte adhesion. J Biol Chem 2007;282:467-77.

21. Michaud JL, Lemieux LI, Dubé M, Vanderhyden BC, Robertson SJ, Kennedy CR. Focal and segmental glomerulosclerosis in mice with podocyte-specific expression of mutant alpha-actinin-4. J Am Soc Nephrol 2003;14:1200-11.

22. DeMali KA, Wennerberg $\mathrm{K}$, Burridge $\mathrm{K}$. Integrin signaling to the actin cytoskeleton. Curr Opin Cell Biol 2003;15:572-82.

23. Shankland SJ, Pippin JW, Reiser J, Mundel P. Podocytes in culture: past, present, and future. Kidney Int 2007;72:26-36. 